\title{
A study of the EAS Cherenkov light time profile with the HiSCORE experiment
}

\author{
Vladimir Samoliga* \\ Irkutsk State University, K.Marx St. 1, 664003 Irkutsk, Russian Federation \\ E-mail: samoligavs@gmail.com
}

The HiSCORE experiment is an array of wide-angle, non-imaging detectors of atmospheric Cherenkov component of EAS operating in the ultra high energy range. Whereas arrays of particle detectors use shower front of secondary particles that reach the observation level, HiSCORE deals with the shower light front, i.e. temporal and spatial distributions of Cherenkov photons generated in EAS. The temporal difference between light fronts of gamma and proton induced EAS is analyzed using Monte Carlo simulations. A gamma-hadron separation possibility based on this difference is studied taking into account realistic detector resolutions of event reconstruction.

35th International Cosmic Ray Conference - ICRC2017

12-20 July, 2017

Bexco, Busan, Korea

\footnotetext{
* Speaker.
} 


\section{Introduction}

A number of questions of cosmology and the particle physics can be solved by studying cosmic rays of ultra high energies $(>30 \mathrm{TeV})$. To search for sources of such cosmic rays - the so-called PeVatrons - it is necessary to determine the direction from which the particles come, but the charged particles move along curved trajectories due to the action of galactic magnetic fields. Information about the direction to the source is carried by electrically neutral particles - neutrinos and photons, including high energetic - gamma quanta. For this reason gamma-ray astronomy began its intensive development at the end of the 20th century and continues to develop actively.

Existing astrophysical installations and gamma-ray astronomy methods covered the energy range from tens of $\mathrm{GeV}$ to about $50 \mathrm{TeV}$. To investigate gamma radiation of energies below 100 $\mathrm{GeV}$ detectors placed on satellites are used. Due to the energy increase the flux of primary particles decreases. In energy range of hundreds $\mathrm{GeV}$ ground-based imaging atmospheric Cherenkov telescopes (IACTs) are used to detect cascades of secondary particles. In such telescopes the Cherenkov image of an extensive air shower (EAS) is recorded using multichannel cameras based on photomultipliers and is analyzed with methods that provide good gamma-hadron separation.

Above $50 \mathrm{TeV}$ the flux is so small that more collection area and observation time are required to get sufficient number of events. Consequently for this energy range an approach was proposed that is used for studying of cosmic rays - an array of detectors covering an area of the order of square kilometers. One of such facilities is TAIGA-HiSCORE, part of the hybrid astrophysical observatory TAIGA [1].

\section{TAIGA-HiSCORE}

TAIGA-HiSCORE is a ground-based wide-angle detector of air shower Cerenkov radiation. Array of optical stations distributed in perspective over an area of $5 \mathrm{~km}^{2}$. It uses the spatial and temporal distributions of Cherenkov photons and is designed for registration of gamma quanta and cosmic rays with energies of $10 \mathrm{TeV}-1 \mathrm{EeV}$ [2]. The field of view of the installation is about $1 \mathrm{sr}$ and allows exploring a vast area of the sky. Due to the large area and the operating mode in multi- $\mathrm{TeV}$ energy range, it is possible to detect primary charged particles in the transition region between the Galactic and extragalactic components of the cosmic-ray spectrum.

Each optical station of the detector includes four 8-inch PMTs equipped with Winston cones, and an electronics unit. Four channels on the one hand provide suppression of false triggers, and on the other hand increase the area of the light collection on the photomultipliers. Thereby light collection area is about $0.5 \mathrm{~m}^{2}$. The station detects an event if the sum of the signals of the four channels exceeds the threshold of 100 photoelectrons per nanosecond. To detect an event, at least four neighboring stations must be triggered. As a response of the station to an event a pulse is generated. The parameters of number of pulses are used to calculate the position of the shower axis, the energy of the primary particle and the depth of the shower maximum.

In this paper the time profile of Cherenkov emission is under investigation. The HiSCORE (63 stations) was modelled using the detector simulation program sim_score++ [3] and EAS simulations were performed in the energy range $30-500 \mathrm{TeV}$ using the CORSIKA package [4]. 


\section{Analysis of the profile}

In [5] the arrival time model was developed which uses measured light arrival times to reconstruct the direction of origin. This model assumes detector position in plane of observation level relatively to shower core and can derive the height of the shower maximum. In this paper the questions are how arrival time of Cherenkov photons depends on the angle between the shower axis and the direction to a detector and is there difference between temporal profiles for gamma and proton induced showers.

For calculations the shower parameters known from Monte-Carlo simulations are used: coordinates of shower core $\left(X_{\text {core }}, Y_{\text {core }}, Z_{\text {core }}\right)$, depth of shower maximum Depth, direction of origin $(\theta, \phi)$. Coordinates of shower maximum are derived as follows:

$$
\begin{array}{r}
Z_{\text {max }}=H_{\text {max }}-H_{0} \\
X_{\text {max }}=Z_{\text {max }} \tan \theta \cos \phi \\
Y_{\text {max }}=Z_{\text {max }} \tan \theta \sin \phi
\end{array}
$$

where $H_{0}$ is height of observation level (675 $\mathrm{m}$ a.s.l.) and $H_{\max }$ is height of shower maximum which is depends on Depth as

$$
H_{\max }=-7652.3 \ln \left(\frac{\text { Depth }+50.4129}{1117.85}\right)
$$

Formula (3.2) is a fit obtained from model of Central European atmosphere AT223 [4] for the heights below $10 \mathrm{~km}$.

Using shower maximum as reference point vectors to the core position $\overrightarrow{\mathbf{A}}$ and to some station $\overrightarrow{\mathbf{B}}$ can be determined as

$$
\overrightarrow{\mathbf{A}}=\left(\begin{array}{c}
X_{\max }-X_{\text {core }} \\
Y_{\max }-Y_{\text {core }} \\
Z_{\max }-Z_{\text {core }}
\end{array}\right), \overrightarrow{\mathbf{B}}=\left(\begin{array}{c}
X_{\max }-X_{s t} \\
Y_{\max }-Y_{s t} \\
Z_{\max }-Z_{s t}
\end{array}\right)
$$

Then the angular distance of the station is:

$$
\alpha=\arccos \frac{(\overrightarrow{\mathbf{A}} \cdot \overrightarrow{\mathbf{B}})}{|\overrightarrow{\mathbf{A}}||\overrightarrow{\mathbf{B}}|}
$$

Here and after the front is supposed to be spherical in the first approximation (figure 1). Then $R_{0}=|\overrightarrow{\mathbf{A}}|$ - radius of a sphere with center in the shower maximum and angular distance can be transformed into linear distance, which is more appropriate - distance from core position, perpendicular to the shower axis:

$$
R_{a x}=R_{0} \tan \alpha
$$

Delay of arrival time for the station includes geometrical part $D$ between sphere and station, propagation medium part $d$ due to the speed of light in the air and shower structure part $\delta$, which is under investigation: 


$$
\text { Delay }=D+d+\delta \Rightarrow \delta=\text { Delay }-D-d
$$

In other words $\delta$ shows how Cherenkov light front differs from spherical front on condition that propagation delay $d$ is discarded.

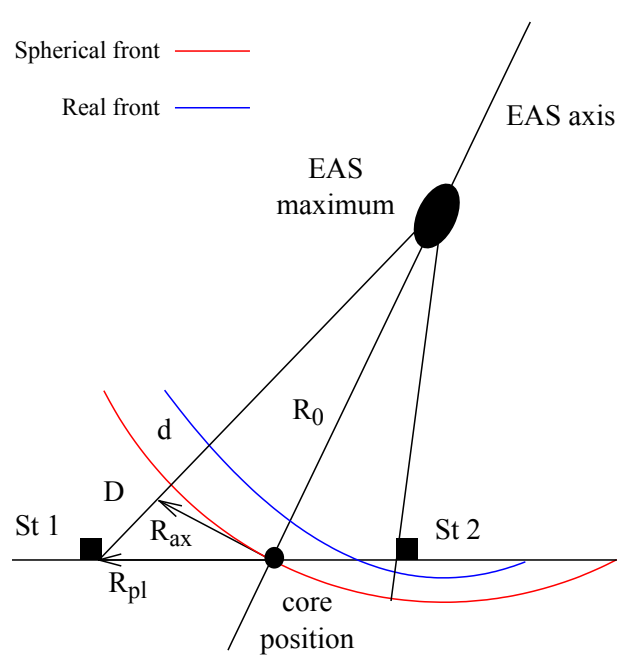

Figure 1: Definition of delays between fronts and geometry of shower and stations positions

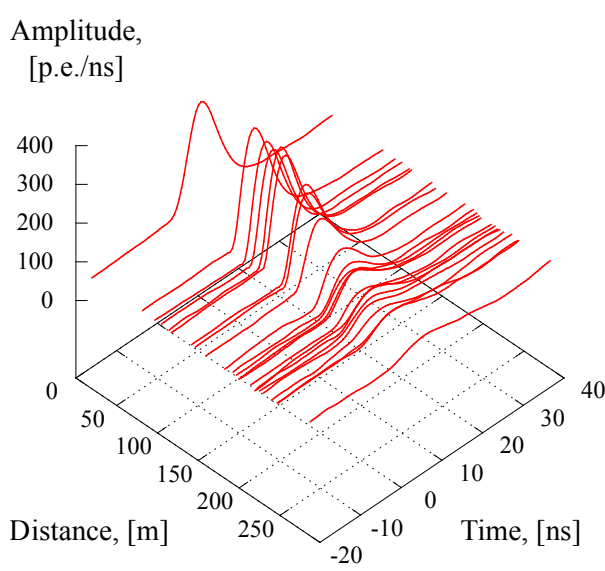

Figure 2: Sorting of the HiSCORE pulses by $R_{a x}$ distance with discarded geometrical delay $D$

Delay is known from simulations and is relative to arrival time at core position $T_{0}=0$. Arrival time of a station is the peak time of the pulse due to assumption that peak time correponds to the shower maximum (figure 2). $D$ is calculated as:

$$
D=\frac{|\overrightarrow{\mathbf{B}}|-|\overrightarrow{\mathbf{A}}|}{C}
$$

where $C=0.299792458 \mathrm{~m} / \mathrm{ns}$ is the speed of light in vacuum.

Propagation time $T_{\text {prop }}$ for the Cherenkov photons that are emitted at altitude $H_{\max }$ and reach the station on the observation level can be calculated as:

$$
T_{\text {prop }}=\int_{H_{0}+Z_{s t}}^{H_{\max }} \frac{d h}{C(h) \cdot \cos (\theta)}
$$

where $\theta$ is angle between zenith and vector $\overrightarrow{\mathbf{B}}$. Speed of light depends on the height $h$ in the atmosphere and can be expressed as:

$$
C(h)=\frac{C}{n(h)}=\frac{C}{1+N(h)}
$$

where index $N(h)=n-1$ can be fitted also using atmosphere model AT223:

$$
N(h)=10^{-6} A \cdot B^{h}
$$

where $A=301.593, B=0.891084$ and $h$ must be taken in $\mathrm{km}$. Then propagation time is: 


$$
T_{\text {prop }}=\frac{1}{C \cdot \cos (\theta)} \int_{H_{0}+Z_{s t}}^{H_{\max }}\left(1+10^{-6} A \cdot B^{h}\right) d h=T_{v a c}+\frac{1}{C \cdot \cos (\theta)} \int_{H_{0}+Z_{s t}}^{H_{\max }} 10^{-6} A \cdot B^{h} d h
$$

where $T_{v a c}$ is propagation time in vacuum and last member is the delay $d$ due to the air:

$$
d=\frac{10^{-3} A}{C \cdot \cos (\theta)} \cdot \frac{B^{H_{\max }}-B^{H_{0}+Z_{s t}}}{\ln (B)}
$$

For example, for photons that move from $8 \mathrm{~km}$ a.s.l. to $675 \mathrm{~m}$ a.s.l. delay $d=4.6 \mathrm{~ns}$.

\section{Results}

Using (3.6) and definitions of delays (3.7) and (3.12) temporal distribution of Cherenkov photons was analyzed for various energies of primary particles and depth of shower maximum. Taking into account geographical latitude of the TAIGA experiment $\left(51^{\circ} 48^{\prime} 32^{\prime \prime}\right)$ few source directions were applied that are placed on the Crab Nebula trajectory in the experiment coordinate system: $\left(\theta=29.66^{\circ}, \phi=0^{\circ}\right),\left(\theta=29.91^{\circ}, \phi= \pm 9.33^{\circ}\right),\left(\theta=30.66^{\circ}, \phi= \pm 18.41^{\circ}\right)$.

For each sort (gamma or proton and energy) of primary particles all of these directions give the same temporal distributions. Hence events from all the directions were processed jointly for each energy level for both types of primary particles. Figure 3 shows distribution of $\delta$ for protons with various energies at the same Depth: with energy increase Cherenkov photons cover more area saving the same characteristic of temporal distribution.

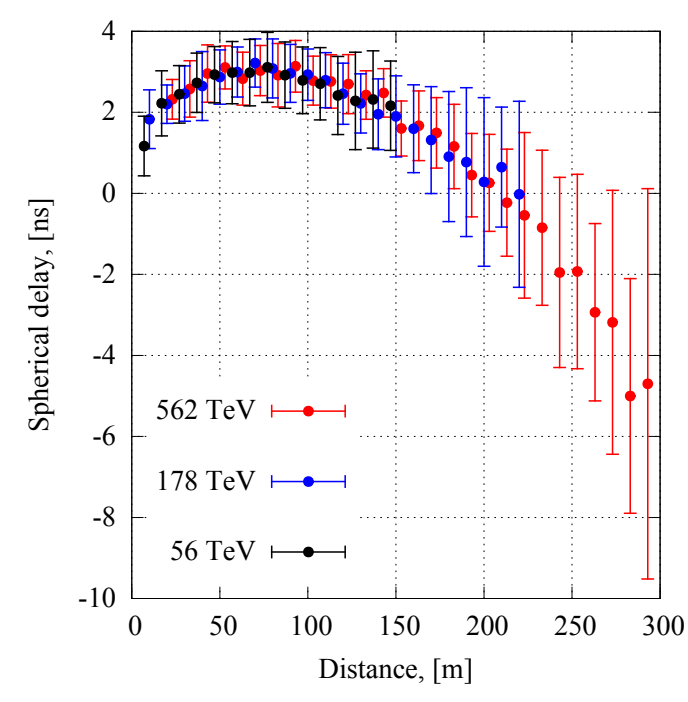

Figure 3: Profiles of protons with various energies and the same Depth=600 $\mathrm{g} / \mathrm{cm}^{2}$

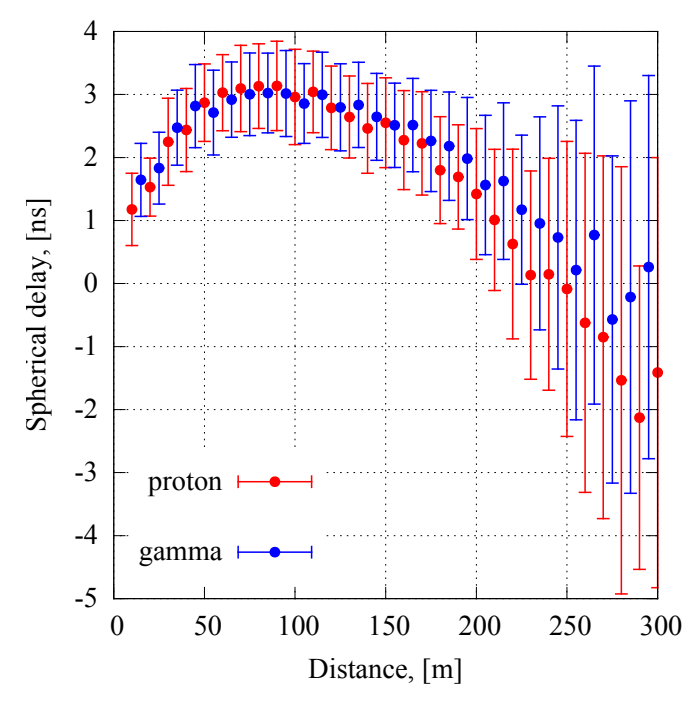

Figure 4: Comparison of gamma $(E=316 \mathrm{TeV})$ and proton $(E=562 \mathrm{TeV})$ induced profiles with the same Depth=550 $\mathrm{g} / \mathrm{cm}^{2}$

Fronts of gamma and proton induced showers were compared for different energy levels and values of Depth. They are almost identical, there is only insignificant difference at large distance from shower axis for high energetic events. For example, in figure 4 proton induced showers have less values of $\delta$ than gamma induced ones for axial distances more than $200 \mathrm{~m}$. 
Taking into account realistic detector resolutions of event reconstruction, especially core position resolution $\sim 7 \mathrm{~m}$ ( $68 \%$ containment) at $\mathrm{E}=500 \mathrm{TeV}$, it is impossible to distinguish the temporal difference in conditions of real experiment.

Yet another result is shown in figure 5: the less value of Depth the more spherical front is. It is logical because event with large altitude of shower maximum looks more compact than event with maximum close to observation level. Negative values of spherical delay for events with large Depth mean that the majority of photons that are detected by further stations are emitted lower than shower maximum.

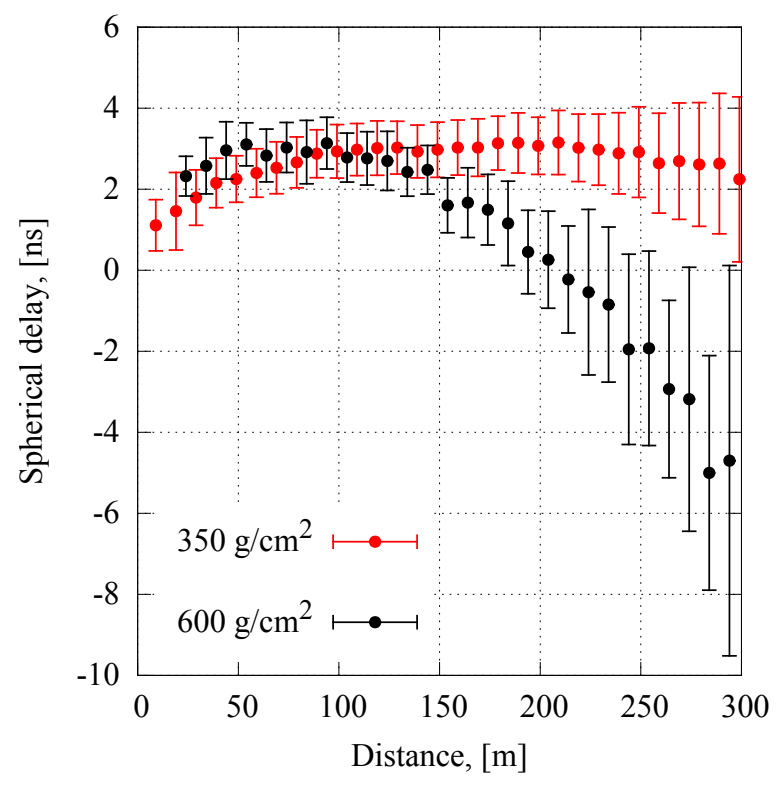

Figure 5: Changing of the profile depending on Depth

\section{Conclusion}

Significant difference between gamma and proton induced temporal profiles was not revealed. For the real experiment with given resolutions of reconstruction the fronts are the same. In [6] a possibility was discussed to detect Cherenkov photons that are produced by secondary hadrons lower than shower maximum and reach the observation level few ns before the main light front. This possibility was checked for HiSCORE in [5] using rize time of the pulses and as a result distributions overlap considerably.

Information about spherical delay at large distances for events with Depth $>400 \mathrm{~g} / \mathrm{cm}^{2}$ can be used for modification of event reconstruction.

\section{Acknowledgements}

This work was supported in part of the Russian Federation Ministry of Education and Science (projects 14.B25.31.0010, 2017-14-595-0001, 3.9678.2017/8.9, 3.904.2017/4.6, 3.6787.2017/7.8, 
1.6790.2017/7.8), the Russian Foundation for Basic Research (grants 16-02-00738, 16-32-00329, 17-02-00905) and the grant 15-12-20022 of the Russian Science Foundation.

Calculations were performed on HPC-cluster «Academician V.M. Matrosov» [7]

\section{References}

[1] N. Budnev et al., The TAIGA experiment: From cosmic-ray to gamma-ray astronomy in the Tunka valley Nucl. Inst. Meth. in Phys. Res. A 845 (2017) 330-333

[2] M. Tluczykont et al., The HiSCORE concept for gamma-ray and cosmic-ray astrophysics beyond 10 TeV, Astropart. Phys. 56 (2014) 42-53 [1403.5688].

[3] M. Tluczykont, et al., The ground-based large-area wide-angle gamma-ray and cosmic-ray experiment HiSCORE, Advances in Space Research 48 (2011) 1935-1941

[4] D. Heck et al., Report FZKA 6019 (1998), Forschungszentrum Karlsruhe; http://www-ik.fzk.de/corsika/physics description/corsika phys.html

[5] D. Hampf, M. Tluczykont, D. Horns, Event reconstruction techniques for the wide-angle air Cherenkov detector HiSCORE Nucl. Inst. Meth. in Phys. Res. A 712 (2013) 137-146 [1302.3957]

[6] H. Cabot et al., Measurable difference in Cherenkov light between gamma and hadron induced EAS, Astropart.Phys. 9 (1998) 269-276

[7] Irkutsk Supercomputer Center of SB RAS, http://hpc.icc.ru 